Mônica Cristina Riccio Ribeiro

Modelagem termodinâmica da deposição de filmes finos de CdTe pela co-evaporação dos elementos, em condições de transporte isotérmico

Tese de Doutorado

Tese apresentada como requisito parcial para obtenção do título de Doutor pelo Programa de Pós-Graduação em Engenharia Metalúrgica do Departamento de Ciência dos Materiais e Metalurgia da PUC-Rio.

Orientador: Roberto Ribeiro de Avillez Co-orientadora: Leila Rosa de Oliveira Cruz

Rio de Janeiro, abril de 2005. 
Monica Cristina Riccio Ribeiro

\title{
Modelagem termodinâmica da deposição de filmes finos de CdTe pela co-evaporação dos elementos, em condições de transporte isotérmico
}

\begin{abstract}
Tese apresentada como requisito parcial para obtenção do grau de Doutor em Engenharia Metalúrgica e de Materiais pelo Programa de Pós-Graduação em Engenharia Metalúrgica do Departamento de Ciência dos Materiais e Metalurgia da PUC-Rio. Aprovada pela Comissão Examinadora abaixo assinada.
\end{abstract}

Prof. Roberto Ribeiro de Avillez Orientador

Departamento de Ciência dos Materiais e Metalurgia - PUC - Rio

Profa. Leila Rosa de Oliveira Cruz

Co-orientadora

Instituto Militar de Engenharia - IME

Prof. Fernando Cosme Rizzo Assunção Departamento de Ciência dos Materiais e Metalurgia - PUC - Rio

Prof. Carlos Luiz Ferreira Instituto Militar de Engenharia - IME

Prof. Sidnei Paciornik Departamento de Ciência dos Materiais e Metalurgia- PUC - Rio

Prof. Sergio Álvaro de Souza Camargo Junior Universidade Federal do Rio de Janeiro - UFRJ

Prof. José Eugenio Leal Coordenador Setorial de Pós-Graduação do Centro Técnico Científico da PUC-Rio 
Todos os direitos reservados. É proibida a reprodução total ou parcial do trabalho sem autorização do autor, do orientador e da universidade.

\section{Mônica Cristina Riccio Ribeiro}

Graduou-se em Engenharia Metalúrgica na UFRJ (Universidade Federal do Rio de Janeiro) em 1990. Obteve o título de Mestre em Engenharia Metalúrgica e de Materiais pelo Programa de Pós-Graduação em Engenharia Metalúrgica do Departamento de Ciência dos Materiais e Metalurgia da PUC-Rio.

Ficha Catalográfica

Ribeiro, Mônica Cristina Riccio

Modelagem termodinâmica da deposição de filmes finos de $\mathrm{CdTe}$ pela coevaporação dos elementos, em condições de transporte isotérmico / Mônica Cristina Riccio Ribeiro ; orientadores: Roberto Ribeiro de Avillez, Leila Rosa de Oliveira Cruz. - Rio de Janeiro : PUC, Departamento de Ciência dos Materiais e Metalurgia, 2005.

114 f. : il. ; $30 \mathrm{~cm}$

Tese (doutorado) - Pontifícia Universidade Católica do Rio de Janeiro, Departamento de Ciência dos Materiais e Metalurgia.

CDD: 669 


\section{Dedicatória}

Dedico este trabalho aos meus filhos, Karina e Raphael, que me ensinaram a ver a vida de um jeito especial. 


\section{Agradecimentos}

A elaboração de uma tese de doutorado é um produto coletivo embora sua redação, responsabilidade e stress sejam predominantemente individuais. Várias pessoas contribuíram para que este trabalho chegasse ao fim. A todas elas registro minha gratidão.

Ao meu orientador Avillez pela sua disponibilidade irrestrita, sua forma exigente, crítica e criativa de argüir as idéias apresentadas, creio que deram norte a este trabalho, facilitando o alcance de seus objetivos. Ao longo destes anos de doutorado, tive a oportunidade e o privilégio de renovar meus conhecimentos e vivenciar novas situações, que se tornaram lições valiosas para toda uma vida. Ao amigo Avillez agradeço a confiança no meu trabalho.

Ao Conselho Nacional de Pesquisa - CNPq - que me concedeu uma bolsa durante a realização deste doutorado, fato este que muito contribuiu para viabilização desta tese.

Ao pessoal da secretaria do DCMM, que em clima de camaradagem e solidariedade, amenizou as intermináveis, e nem sempre proveitosas, horas em que fiquei no departamento ao longo do estudo. À Luzinete e Amarildo, que fizeram com que este trabalho se concretizasse de forma a observar as normas acadêmicas vigentes, também expresso meus agradecimentos.

Aos profissionais que fizeram essa tese comigo: Ronaldo Pedro da Silva (Raios-X), Maurício Monteiro (MEV), Marcos Henrique (LPDI), Henrique Duarte Filho (AFM) pela sua contribuição na análise de dados obtidos neste trabalho, sem a qual não terminaria essa tese.

A meus amigos que compreenderam a divisão meio tumultuada de tempo e atenção entre meus próprios estudos e a amizade.

Aos meus pais, pela sólida formação dada até minha juventude, que me proporcionou a continuidade nos estudos até a chegada a este doutorado, meus agradecimentos. 
Um agradecimento muito especial a meu marido por tolerar minhas ausências e pela ajuda tão necessária.

Aos meus filhos Karina e Raphael que, como disse Mário Prata, ameaçavam:

“- Não vou mais estudar! Não vou mais na escola.

- Quero estudar mais, não. Olha vocês dois. Não fazem mais nada na vida. É só a tese, a tese, a tese. Não pode comprar bicicleta por causa da tese. A gente não pode ir para a praia por causa da tese. Tudo é pra quando acabar a tese. Até trocar o pano do sofá. Se eu estudar vou acabar numa tese. Quero estudar mais, não.

Não me deixam nem mexer mais no computador. Vocês acham mesmo que eu vou deletar a tese de vocês?

Pensando bem, até que não é uma má idéia!"

A minha sogra Dulce por ter sido mãe dos meus filhos tantas vezes. Sua dedicação e ajuda foi imprescindível para o término desse trabalho

A todos os que ficam exultantes com os meus "feitos" e torcem por minhas pequenas e sofridas conquistas, que, para eles, são sempre grandes vitórias. 


\section{Resumo}

Riccio Ribeiro, Mônica Cristina. Modelagem termodinâmica da deposição de filmes finos de CdTe pela co-evaporação dos elementos, em condições de transporte isotérmico. Rio de Janeiro, 2005. 114p. Tese de Doutorado Departamento de Ciência dos Materiais e Metalurgia, Pontifícia Universidade Católica do Rio de Janeiro.

O objetivo do presente trabalho é a deposição de filmes de telureto de cádmio a partir de duas fontes de materiais, $\mathrm{Cd}$ e Te, com base no uso de diagramas de potenciais termodinâmicos para avaliar as condições de deposição. Em especial, o método proposto permite avaliar a influência de contaminantes gasosos, tais como, oxigênio, sobre as fases condensadas. O método também pode ser aplicado para a deposição de outros compostos que sejam mais estáveis que os elementos que os compõem. O processamento utilizado na deposição utiliza uma técnica alternativa onde as temperaturas de fonte e de substrato são as mesmas.

\section{Palavras-chave}

filme fino; termodinâmica computacional; processo de deposição; telureto de cádmio 


\section{Abstract}

Riccio Ribeiro, Mônica Cristina. Thermodynamic modelling of CdTe thin film deposition by elemental co-evaporation, under isothermal transport. Rio de Janeiro, 2005. 114p. DSC Thesis - Departamento de Ciência dos Materiais e Metalurgia, Pontifícia Universidade Católica do Rio de Janeiro.

The objective of the present work is deposition of Cadmiun Telurides films from two sources of materials, $\mathrm{Cd}$ and $\mathrm{Te}$, on the basis of the use of diagrams of thermodynamic potentials to evaluate the deposition conditions. In special, the considered method allows to evaluate the influence of gaseous contaminantes, such as, oxygen, on the condensed phases. The method can be applied for the deposition of other compounds that are more stable than the constituent elements. The equipment used in the deposition uses an alternative technique where the temperatures of source and substrate are the same ones.

\section{Keywords}

thin film; computational thermodynamics; deposition process; cadmiun telluride 


\section{Sumário}

1 Introdução 15

1.1. Motivação 15

1.2. Objetivos 20

2 Revisão Bibliográfica $\quad 22$

2.1. Células solares de CdTe 22

2.1.1. Histórico 22

2.1.2. Estrutura 23

2.2. Propriedades do CdTe 25

2.3. Deposição de filmes finos por evaporação 30

2.3.1. Nucleação e crescimento 32

2.3.2. Co-deposição 36

2.3.3. Influência de impurezas no processo de deposição 37

2.3.4. A cinética de crescimento de cristais 39

2.3.5. Morfologia de filme e densidade 41

2.4. Técnica de sublimação em espaço reduzido (CSS) para deposição de filmes de CdTe 42

2.5. Conceitos da teoria cinética 44

2.5.1. Vaporização 45

2.5.1.1. Distribuição do vapor 46

2.5.1.2. Livre caminho médio $\quad 47$

2.5.1.3. Taxa de evaporação 49

$\begin{array}{ll}\text { Fluxo molecular } & 49\end{array}$

$\begin{array}{ll}\text { Fluxo viscoso } & 50\end{array}$ 
2.5.2. Modelo de fluxo de difusão 51

2.5.2.1. Fluxo molecular 51

2.5.2.2. Fluxo viscoso 53

2.5.2.3. Velocidade 54

2.5.2.4. Tempo de formação de uma monocamada de $\mathrm{Cd}$ e $\mathrm{Te}_{2}$. $\quad 55$

2.5.3. Modelo de fluxo total do gás 56

2.5.4. Gás residual (oxigênio) e taxas de transporte de massa 60

3 Resultados 63

3.1. Base de dados termodinâmicos 63

3.1.1. Reações com o $\mathrm{CdO}(\mathrm{s})$ e $\mathrm{Te}_{\mathrm{x}} \mathrm{O}_{\mathrm{y}}(\mathrm{s}) \quad 64$

3.1.1.1. Reações químicas 65

3.1.2. Reação de formação do CdTe sem a presença de gases residuais66

3.2. Modelagem termodinâmica 69

3.2.1. Introdução 69

3.2.2. Diagrama de fases $\quad 70$

3.2.3. Pressão de vapor $\quad 71$

3.2.4. Diagrama de predominância $\quad 74$

3.3. Método de deposição proposto: CSS diferenciado 78

3.3.1. Introdução 78

3.3.2. Sistema e processo de deposição 80

3.3.2.1. Equipamento de deposição 81

3.3.2.2. Cadinho de grafite $\quad 84$

3.3.2.3. Substrato $\quad 86$

3.4. Validação do método proposto $\quad 87$

3.4.1. Microscópio Eletrônico de Varredura (MEV) 87

3.4.1.1. Medidas de Espessuras $\quad 87$

3.4.1.2. Tamanho de grão 92

3.4.2. Difração de Raios-X 94

3.4.2.1. Análise dos espectros de raios-X 96

3.4.3. Microscópio de Força Atômica 99 


\section{Lista de figuras}

Figura 1 - Desenvolvimento do mercado mundial de fotovoltaicos em MWp[1].

Figura 2 - Mercado mundial de fotovoltaicos de diferentes materiais semicondutores em 1998 [1].

Figura 3 - Eficiência de conversão de materiais de células solares versus banda proibida para células de homojunção [1].

Figura 4 - Seqüência de filmes normalmente utilizada na célula solar a filme fino $\mathrm{CdS} / \mathrm{CdTe}$ ("backwall").

Figura 5 - Tabela periódica mostrando os elementos dos grupos II e VI 26

Figura 6 - Estrutura esfalerita

Figura 7 - Estrutura wurtzita hexagonal

Figura 8 - Diagramas esquemáticos das estruturas (a) hexagonal e (b) cúbica do CdTe. Em a, a orientação é tal que o plano basal do hexagonal compacto é horizontal e a seqüência $\mathrm{AB}-\mathrm{AB}-\mathrm{AB}$ é destacada. Em b, a orientação é tal que o plano compacto 111 é horizontal e a seqüência $\mathrm{ABC}-\mathrm{ABC}-\mathrm{ABC}$ é destacada. As estruturas das células unitárias estão sobrepostas nos diagramas [13].

Figura 9 -Variação de entalpia do CdTe a 0K para as estruturas esfalerita e wurtzita como função da pressão [12].

Figura 10 - A energia interna (eV/atom) do sistema como função da temperatura para o CdTe [12].

Figura 11 - O parâmetro de rede como função da temperatura para o CdTe na estrutura esfalerita [12].

Figura 12 - Esquema de deposição de filmes finos

Figura 13 - Mecanismo possível de deposição de CdTe - mecanismo de Eley-Rideal. Lado esquerdo: átomos de $\mathrm{Cd}(\mathrm{g})$ reagem com Te condensado para formar CdTe antes que o Te possa re-evaporar. $\mathrm{O}$ processo análogo - com $\mathrm{Te}_{2}(\mathrm{~g})$ reagindo com $\mathrm{Cd}$ momentaneamente condensado, é indicado no lado direito [19].

Figura 14 - Ilustração esquemática dos modos de crescimento de (1) Volmer-Weber (VW) - crescimento 3D de ilhas, (2) Frank-van der Merwe (FM) - modo de crescimento 2D 
de camadas e (3) Stranski-Krastanov - modo composto de ilhas e camadas.

Figura 15 - Figuras esquemáticas de estruturas compostas no caso de (a) baixa e (b) alta concentração de componentes aditivos minoritários, e no caso de aditivos como o maior componente (c) [30].

Figura 16 - Impurezas podem melhorar (a) ou inibir (b) a molhabilidade [29]

Figura 17 - Desenho esquemático de um elemento superficial recebendo cobertura de uma fonte de pequena área

Figura 18 - Distribuição de vapores 45

Figura 19 - Geometria da câmara de evaporação 46

Figura 20 - Fluxo de átomos de cádmio e de telúrio em função da temperatura de evaporação.

Figura 21 - Tempo de formação de uma monocamada em relação a temperatura. $\quad 56$

Figura $22-\mathrm{P}_{\mathrm{Cd} / \mathrm{CdTe}}$ e $P_{\mathrm{Te}_{2} / C d T e}$ em função da temperatura 58

Figura 23 - Variação de espessura do filme ao longo do substrato. 60

Figura 24 - Dados termodinâmicos obtidos a partir do programa Thermocalc [60] 67

Figura 25 - (a) Diagrama de predominância para as condições da deposição $T=773 \mathrm{~K}$ e $\mathrm{P}=0.1 \mathrm{~Pa} \approx 10^{-3}$ mbar. (b) Diagrama PxT mostrando as fases mais estáveis. $\quad 68$

Figura 26 - Diagrama de fases Cd-Te para pressão atmosférica [68] 71

Figura 27 - Pressões de vapor de $\mathrm{Cd}, \mathrm{Te}_{2}$, e $\mathrm{CdTe}$ em equilíbrio com suas fases condensadas em função da temperatura.

Figura 28 - Pressão de vapor de Cd/CdTe, Te/CdTe e CdTe/CdTe 73

Figura 29 - Diagramas de potencial do gás oxigênio versus o potencial das espécies gasosas $\mathrm{Te}_{2}$ e $\mathrm{Cd}$ mostrando as fases presentes: $\square$ gás, $\square \mathrm{CdO}, \square \mathrm{TeO}_{2}, \square \mathrm{CdTe}$. As setas representam o aumento da pressão na câmara de reação. 76

Figura 30 - Diagramas de potencial do gás oxigênio versus o potencial das espécies gasosas $\mathrm{Te}_{2}$ e $\mathrm{Cd}$ mostrando as fases presentes: $\square$ gás, $\square \mathrm{CdO}, \square \mathrm{TeO}_{2}, \square \mathrm{CdTe}$. As setas representam o aumento da temperatura $(600 \mathrm{~K} \rightarrow 700 \mathrm{~K} \rightarrow 800 \mathrm{~K})$ para uma mesma pressão de $0.1 \mathrm{~Pa}$ na câmara de reação.

Figura 31 - Esquema do sistema de deposição onde $\square$ aço inoxidável 304, $\square$ termopar, substrato de vidro, cola, $\square$ cadinho de grafite, $\mathbf{\square}$ forno tubo de quartzo 81

Figura 32 - Temperatura do forno em função do tempo para a amostra depositada durante 
$10 \min (a)$ e durante $2 \min (b)$.

Figura 33 - Cadinho de grafite apresentando 3 sulcos para colocação de materiais fontes.

Pedaços de cádmio e pós de telúrio eram colocados sempre nas laterais, deixando o sulco do meio livre.

Figura 34 - Vistas laterais dos cadinhos (a) Configuração do cadinho para D20, D21 e D22 e (b) Configuração do cadinho para D23.

Figura 35 - Desenho esquemático da posição das fontes em relação ao substrato

Figura 36 - Perfil de fluxos para Cd e Te ao longo de um substrato de $27 \mathrm{~mm}$ de comprimento

Figura 37- (a) Perfil de deposição para o CdTe. (b) Ampliação do gráfico (a) para observação do fluxo viscoso

Figura 38 - Micrografias SE obtidas no MEV: D20 (x3000), D21 (x3000), D22 (x5000) e D23 (x5000)

Figura 39 - Micrografias SE obtidas no MEV: D20 (x10000), D21 (x10000) e D22 (x5000) e D23(x30000)

Figura 40 - Espectro de raio-X das amostras de CdTe 96

Figura 41- Micrografias obtidas por AFM para deposição D20 101

Figura 42- Micrografias obtidas por AFM para deposição D21 102

Figura 43- Micrografias obtidas por AFM para deposição D22 103

Figura 44- Micrografias obtidas por AFM para deposição D23 104 